\title{
Intervenções Realizadas no Ambiente de Trabalho para o Uso de Drogas: Revisão Sistemática
}

Interventions Carried Out in the Workplace for the Use of Drugs: Systematic Review

Las Intervenciones Realizadas en el Lugar de Trabajo para el Uso de Drogas: Revisión Sistemática

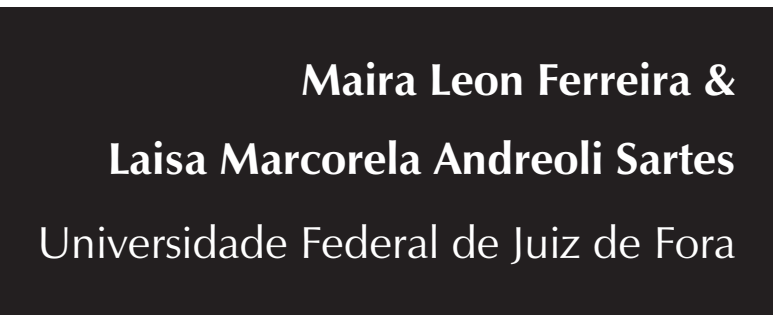

http://dx.doi.org/10.1590/1982-3703001802013 
Resumo: $\mathrm{O}$ uso abusivo de drogas no ambiente de trabalho gera consequências como absenteísmo, baixa produtividade, aumento de custos para empregados e empregadores, piora na saúde do indivíduo e acidentes de trabalho. Objetivo: Realizar uma revisão sistemática de artigos científicos sobre as intervenções realizadas no ambiente de trabalho para o uso de drogas, publicados no período de 2002 até 2012. Método: Os artigos foram pesquisados em seis bancos de dados: Medline (PUBMED), PsychINFO, Web of Science, Lilacs, PEPSIC e Scielo. Os descritores variaram de acordo com o controle de vocabulário de cada base de dados. Inicialmente foram encontrados 97 artigos que foram classificados em Descritivos; Revisões, Medições e Intervenções. A análise de conteúdo foi aplicada a dez artigos que se tratavam de intervenções realizadas no local de trabalho. Resultados: Todos os artigos da revisão sistemática tiveram resultados positivos quanto a seus objetivos de pesquisa, porém todos tiveram falhas metodológicas. Conclusão: Conclui-se que o conjunto de estudos avaliados ainda é insuficiente de evidências científicas e demandam mais estudos que busquem investigar a validade das intervenções e sua aplicabilidade no contexto de trabalho.

Palavras-chave: Uso de drogas. Ambiente de trabalho. Intervenções. Trabalhadores.

Abstract: The drug abuse in the workplace creates consequences such as absenteeism, low productivity, increased costs for employees and employers; worsening health of the individual and workplace accidents. Objectives: Conduct a systematic review of scientific articles on interventions in the workplace for the use of drugs, published from 2002 until 2012. Method: Articles were searched six databases: Medline (PubMed), PsychINFO, Web of Science, LILACS, and SciELO PEPSIC. The descriptors varied by controlling each vocabulary database. Initially found 97 articles that were ranked Descriptions, Reviews, Measurements and Interventions. The content analysis was applied to ten articles that these were interventions in the workplace. Results: All articles of the systematic review were positive about their research objectives, but all had methodological flaws. Conclusion: We conclude that the set of evaluated studies is still insufficient scientific evidence and demand more studies that seek to investigate the validity of the interventions and their applicability in the work context.

Keywords: Drug use. Workplace interventions. Workers.

Resumen: El uso indebido de drogas en el lugar de trabajo crea consecuencias tales como el ausentismo, la baja productividad, el aumento de los costos para los empleados y los empleadores; empeoramiento de la salud del individuo y de los accidentes de trabajo. Objetivo: Realizar una revisión sistemática de artículos científicos sobre las intervenciones en el lugar de trabajo para el uso de drogas, publicada desde 2002 hasta 2012. Método: Se buscaron seis bases de datos: Medline (PubMed), PsychINFO, Web of Science, LILACS y SciELO PEPSIC. Los descriptores variadas mediante el control de cada base de datos de vocabulario. Inicialmente encontrado 97 artículos que fueron clasificados Descripciones, Opiniones, Mediciones e intervenciones. El análisis de contenido se aplicó a diez artículos que se trataba de las intervenciones en el lugar de trabajo. Resultados: Todos los artículos de la revisión sistemática fueron positivos acerca de sus objetivos de la investigación, pero todos tenían defectos metodológicos. Conclusión: Llegamos a la conclusión de que el conjunto de los estudios evaluados es aún suficiente evidencia científica y exigir más estudios que tratan de investigar la validez de las intervenciones y su aplicabilidad en el contexto laboral.

Palabras clave: El uso de drogas. Las intervenciones en el lugar de trabajo. Trabajadores.

\section{Introdução}

A realidade contemporânea tem colocado novos desafios no campo da saúde, principalmente na área de álcool e outras drogas. O uso abusivo de drogas tornou-se um problema social e uma grande questão de saúde pública. A Organização Mundial da Saúde - OMS (1993) reitera em seus documentos que $70 \%$ dos indivíduos que possuem problemas de abuso de álcool e 63\% dos usuários de outras drogas estão empregados. No Brasil, o Ministério da 
Previdência Social observou um aumento de 24,4\% em afastamentos trabalhistas por "dependência química" entre trabalhadores de várias categorias (Brasil, 2012).

De um modo geral, o uso de drogas sempre esteve presente na história da humanidade, sendo utilizadas em diferentes contextos e com objetivos variados, como festas e comemorações, rituais religiosos, tratamento de doenças, alívio da dor, prazer e como forma de vivência de experiências desconhecidas (Spricigo \& Alencastre, 2004). Observa-se que o fenômeno do uso de drogas está inserido em uma problemática multidimensional, portanto não basta circunscrevê-lo a uma relação reducionista existente entre uma pessoa e uma substância. Faz-se necessário considerar a interação que ambos efetuam num determinado contexto, os valores e crenças que permeiam essa interação e suas relações sociais, econômicas e políticas, incluindo-se o contexto do trabalho (Brasil, 2012).

O mundo do trabalho sofreu nas duas últimas décadas profundas transformações em sua organização. De acordo com Mendonça, Souza e Oliani (2008), diante das transformações econômicas, políticas, sociais e avanços do mundo do trabalho surge a necessidade de um novo tipo de colaborador, ajustado às novas demandas da sociedade trabalhista. Consequentemente, as características necessárias ao trabalhador, para adaptar-se a um novo mercado de trabalho, também passaram a ser bem peculiares.

Segundo Beck e David (2007), o tempo despendido com a obtenção da droga e o próprio tempo sob o seu efeito produzem um quadro de desorganização com diferentes níveis de comprometimento, percebidos como: aumento do absenteísmo no trabalho, dificuldades em cumprir as tarefas/metas estipuladas, perda do emprego, negligência dos papéis sociais, interrupção dos estudos, enfraquecimento das relações familiares, descompromisso financeiro, subordinação a subempregos, desvalorização da autoimagem, traços depressivos e sentimentos envolvendo negatividade.

Dados da OMS esclarecem que o indivíduo com dependência de álcool falta cinco vezes mais ao trabalho e que, ainda, tem uma redução expressiva na produtividade (Brasil, 2012). O abuso de substâncias é reconhecido como um sério risco para a saúde e segurança do usuário e também de seus pares (NIAAA, 1997). No local de trabalho, esse uso é associado com o absenteísmo, cochilos durante as atividades laborais, problemas interpessoais e mal desempenho no trabalho (Mangione et al., 1999; Newcomb, 1988; Normand \& Salyards, 1989).

Os locais de trabalho também parecem ser locais apropriados para a realização de intervenções, porque a maioria das pessoas passa períodos substanciais do tempo dedicando-se às atividades laborais (Roman \& Blum, 1996). No que se refere a intervenções focadas no uso de substâncias no ambiente de trabalho, a Secretaria Nacional de Políticas sobre Drogas - SENAD (Brasil, 2012) recomenda que o foco da atenção não deve ser somente o dependente mas também aqueles que fazem uso abusivo (ou nocivo) de álcool e outras drogas. Dessa forma, a PND defende a execução de programas de prevenção dentro do ambiente de trabalho, o que contribui para elevar os índices internos de saúde e segurança. O mesmo documento menciona que apenas uma pequena parcela das empresas brasileiras tem alguma ação concreta sobre o tema, mas que esse número vem aumentando nos dias atuais. Ainda é citado que em países como os Estados Unidos, Canadá, França e Inglaterra o índice de empresas com programas efetivos de atenção e prevenção ao uso de drogas chega a 90\%, o que demonstra a importância dada ao tema por essas corporações (Brasil, 2012).

Segundo a SENAD, os benefícios que são citados a favor dos programas de prevenção ao uso de álcool e outras drogas somam-se a: redução de despesas com assistência médica; redução do absenteísmo, atrasos e licenças médicas; melhora e/ou manutenção da boa imagem da empresa diante do mercado; aumento da lucratividade e criação de novos empregos; melhora da produtividade e do rendimento dos serviços; redução do custo dos negócios; diminuição na frequência dos problemas disciplinares e negligências no local de trabalho. Também são explícitos os variados tipos de programas de prevenção, nos quais é esclarecido que não existem modelos prontos 
de intervenção e, sim, várias estratégias que devem ser adequadas ao tipo de usuário e à estrutura da organização (Brasil, 2012).

Em uma revisão sistemática recente sobre o uso de álcool no local de trabalho Webb, Shakeshaft, Sanson-Fisher e Havard (2009) identificaram dez estudos dos quais apenas quatro se tratavam de ensaios clínicos randomizados. Apesar de todos os estudos mostrarem várias falhas metodológicas, concluiu-se que intervenções breves, intervenções contidas dentro de exames periódicos de saúde e estilo de vida, capacitação psicossocial e programa de pares podem ter resultados benéficos. Os mesmos autores afirmam que há uma escassez de estudos com intervenções no local de trabalho para a área de álcool e outras drogas.

Nessa perspectiva, o presente estudo pretendeu realizar uma revisão sistemática da literatura acerca das intervenções para álcool e outras drogas realizadas no ambiente de trabalho entre estudos publicados em periódicos nacionais e internacionais entre os anos de 2002 e 2012 indexados em seis bases de dados. Como objetivos específicos buscouse identificar os indicadores bibliométricos e através da análise de conteúdo identificar as principais características dos estudos sobre intervenções.

\section{Método e procedimentos}

A revisão sistemática foi realizada através da consulta a seis bases de dados: Medline (PUBMED), PsychINFO, Web of Science, Lilacs, PEPSIC e Scielo, abrangendo os artigos publicados no período entre 2002 até 2012. A justificativa da escolha das bases de dados se deu pela relação do tema com o conteúdo indexado. Para melhor definição dos termos de busca nas bases selecionadas foram utilizadas palavras-chave indexadas pelas próprias bases através de procedimentos de controle de vocabulário. Portanto, os termos da pesquisa variaram conforme a base de dados, a fim de garantir uma busca mais efetiva dos estudos. Os termos escolhidos para a pesquisa referem-se ao uso de drogas no ambiente de trabalho com a justificativa de não restringir as intervenções realizadas nesse contexto. A escolha desses termos abrange um universo geral do que tem sido publicado sobre o tema. Os resultados de cada um dos termos foram cruzados entre si utilizando-se o operador booleano "AND" com a finalidade de restringir a pesquisa aos resumos que apresentavam ao mesmo tempo cada um dos termos.

Para a pesquisa na Medline, os termos utilizados foram o Substance-Related Disorders and (Workplace). Esses termos foram baseados no Medical Subject Heading Terms (Mesh Terms -MeSH), desenvolvido pela U. S. National Library of Medicine, que é utilizado como método de controle de vocabulário para essa base. A pesquisa foi realizada no idioma inglês por se tratar de uma base de dados onde predomina a língua inglesa.

$\mathrm{Na}$ base de dados Web of Science foram utilizados os termos (substance abuse) and (workplace). Os parênteses foram utilizados com a finalidade de buscar as palavras exatas para essa base. Os termos estão na língua inglesa por se tratar de uma base de dados americana.

Os termos utilizados na base de dados Psyclnfo foram Workplace and (Substance Abuse); Trabalho e (Abuso de Drogas); Trabajo y (Abuso de Drogas). Esses termos foram retirados do Thesaurus of Psychological Index Terms utilizado como método de controle de vocabulário por essa base, tendo sido desenvolvido pela American Psychological Association com a finalidade de classificar e definir brevemente diversos termos utilizados no vocabulário da área. Os termos foram procurados nas línguas inglesa, espanhola e portuguesa por se tratar de uma base latino-americana.

Os termos utilizados para a pesquisa na base LILACS (Literatura Latino Americana e do Caribe em Ciências da Saúde) foram: "Drug abuse" and "Workplace/ "Drugs" and "Workplace"| "Drogas" e "Trabalho"|"Abuso de drogas" y "Trabajo"I "Drogas e Trabajo". Esses termos referem-se aos Descritores em Ciências da Saúde (DeCs) adaptados pela BIREME (Centro Latino-Americano e do Caribe de Informação em Ciências da Saúde) a partir do vocabulário do $\mathrm{MeSH}$.

As bases de dados brasileiras, SciELO (Scientific Eletronic Library Online - Brasil) e PEPsic (Periódicos Eletrônicos em Psicologia) não 
possuíam controle de vocabulário, portanto, a busca foi feita utilizando-se os termos do controle de vocabulário dos DeCs presentes na BVS Psi. Esses descritores foram utilizados a fim de ampliar o critério de busca nas três línguas.

Os descritores de cada base de dados estavam presentes no título e no resumo dos trabalhos. Foram critérios de inclusão: revisões, revisões sistemáticas e artigos empíricos que estivessem nas línguas inglesa, espanhola e portuguesa. Também foram considerados os artigos que mencionaram no título e nos resumos o nome das drogas propriamente dito como: maconha, tabaco, álcool, crack, êxtase, metadona, ópio, ou que se referiram a sinônimos como: "drug use", "drug addiction", "addiction", "intoxicaded workers", "chemical dependency", "drug consumption". Os seguintes sinônimos para "local de trabalho" foram considerados: "employee", "employers", "occupation", "industry", "organizational", "working population", "worksite".

Foram excluídas desta pesquisa as teses e dissertações, livros e capítulo de livros e estudos em que a palavra "trabalho" não se referia ao ambiente de trabalho e ao ato de laborar. Também foram excluídos os trabalhos publicados em idiomas que não inglês, espanhol e português. A Tabela 1 descreve os termos utilizados em cada base:
Após realizada a busca nas bases de dados, foi feito o refinamento a partir dos critérios de exclusão e foram encontrados 549 artigos. A partir da leitura dos resumos dos artigos, foram retirados 14 trabalhos que estavam duplicados e 438 que não satisfaziam os critérios de inclusão. Os artigos encontrados foram exportados para o programa Zotero e os indicadores bibliométricos foram tabulados no Microsoft Excel. Após essa etapa de exclusão dos artigos restaram 97 publicações.

Esses 97 artigos foram, inicialmente, classificados por tipo de pesquisa, conforme apresentado na Figura 1. A categoria Intervenções $(N=19)$ foi escolhida para a análise qualitativa dos dados. A justificativa pela escolha dessa categoria se deu pela necessidade de aprofundamento das metodologias utilizadas para intervenções no ambiente de trabalho. O intuito de aprofundar nas intervenções é averiguar o que tem sido feito no ambiente de trabalho e quais intervenções fornecem resultados mais efetivos nessa área. Sobre os tipos de pesquisa, os 97 artigos foram divididos em Descritivos, Revisões, Medições e Intervenções. Na subcategoria Descritivos foram incluídos os trabalhos com análise de dados qualitativa e analítica. Em Revisões foram inclusos os artigos de Revisões bibliográficas, sistemáticas e bibliométricas. Em Medições foram incluídos os artigos que se referiam a levantamento de dados sobre populações e desenvolvimento de instrumentos de medida.

Tabela 1. Bases de dados e termos empregados

\section{Base de Dados/ Termos Empregados}

Medline (Pubmed): Substance-Related Disorders and (Workplace)

Web of Science: (substance abuse) and (workplace)

PsychINFO: Workplace and (Substance Abuse); Trabalho e (Abuso de Drogas); Trabajo y (Abuso de drogas)

Lilacs: "Drug abuse" and "Workplace"| Drugs":and "Workplace"| "Drogas" e "Trabalho"| "Abuso de drogas" y "Trabajo" /"Drogas e Trabajo"

Pepsic: "Drug abuse" and "Workplace"| Drugs" and "Workplace"| "Drogas" e "Trabalho"| "Abuso de drogas" y "Trabajo" /"Drogas e Trabajo"

Scielo: "Drug abuse" and "Workplace"| Drugs" and "Workplace"|"Drogas" e "Trabalho"| "Abuso de drogas" y "Trabajo" /"Drogas e Trabajo" 


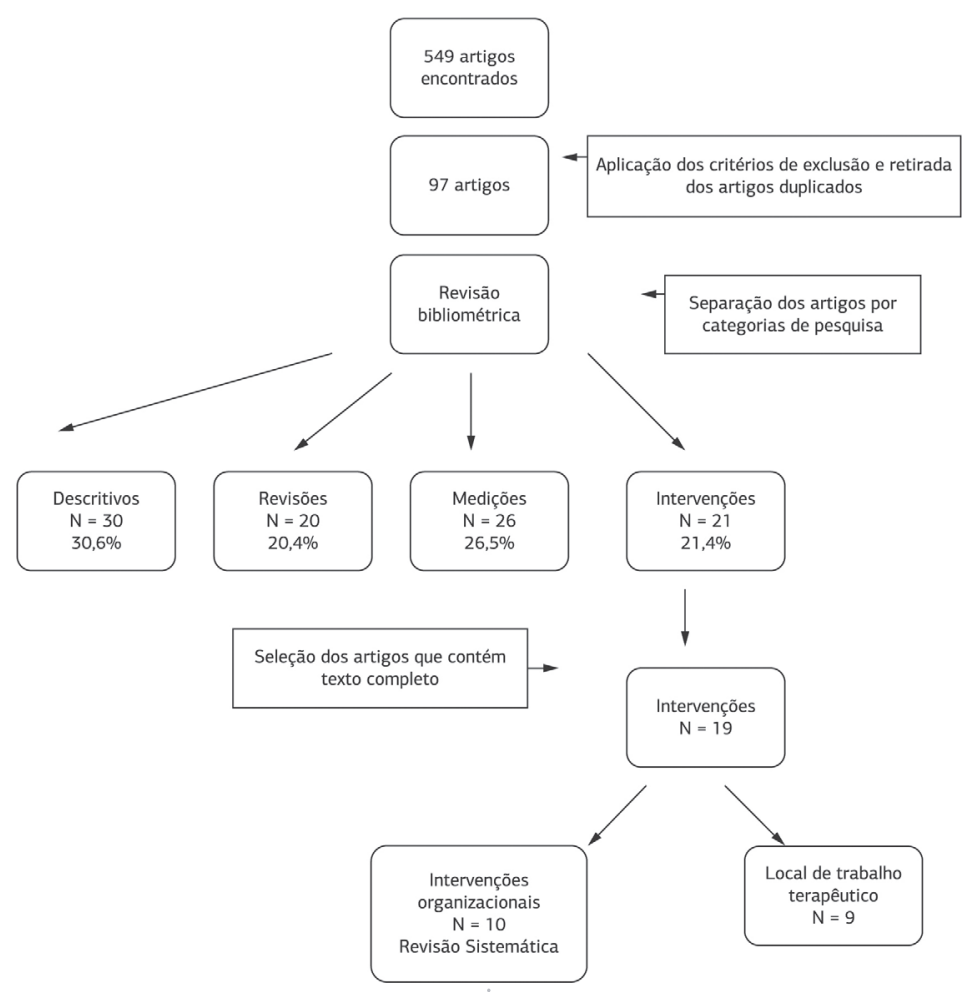

Figura 1. Procedimentos metodológicos utilizados para a coleta dos estudos.

A subcategoria Intervenções se trata de ensaios ou intervenções feitas no local de trabalho para o uso de drogas.

A partir dos artigos que eram intervenções foram escolhidos dez estudos para a revisão sistemática, que se tratavam de intervenções propriamente ditas realizadas no local de trabalho. Os nove artigos restantes não foram incluídos para a análise qualitativa, pois tratavam de um "Local de Trabalho Terapêutico", referindo-se a intervenções voltadas para desempregados e moradores de rua.

Os dez artigos foram analisados pelos seguintes indicadores bibliométricos: autoria, ano de publicação, periódico e idioma de publicação. Logo após procederam-se às análises qualitativas do conteúdo de cada artigo com o propósito de identificar os principais temas abordados nos objetivos, instrumentos utilizados, população de estudo e principais resultados. Esse processo está detalhado na Figura 1. Para a análise qualitativa foi utilizada a Análise de Conteúdo que, segundo Vergara (2005), é utilizada no tratamento de dados que visa identificar o que vem sendo dito acerca de determinado tema.

\section{Resultados}

Indicadores bibliométricos dos artigos da categoria Intervenções

A categoria Intervenções foi analisada pelos seguintes indicadores bibliométricos: idioma, ano de publicação, periódico e autoria, os dados das publicações estão apresentados na Tabela 2. Dos artigos que foram separados para a Revisão sistemática, nove foram publicados em inglês e um em português. Quanto ao ano de publicação, um artigo foi publicado em 2012, um em 2009, um em 2007 e um em 2005; dois artigos foram publicados em 2010, dois em 2008 e dois em 2002. Nenhum artigo foi publicado nos anos de 2003, 2004, 2006 e 2011.

Quanto aos periódicos, dois artigos foram publicados na revista Alcohol and Alcoholism, os demais artigos foram publicados (um em cada) nos periódicos: Drug and Alcohol Review; Psychiatric Services; Journal of Substance Abuse Treatment; Accident Analysis and Prevention; Alcoholism: Clinical and Experimental Research; Health Education Research; Journal of Applied Social Psychology; Psicologia: Ciência e Profissão. 


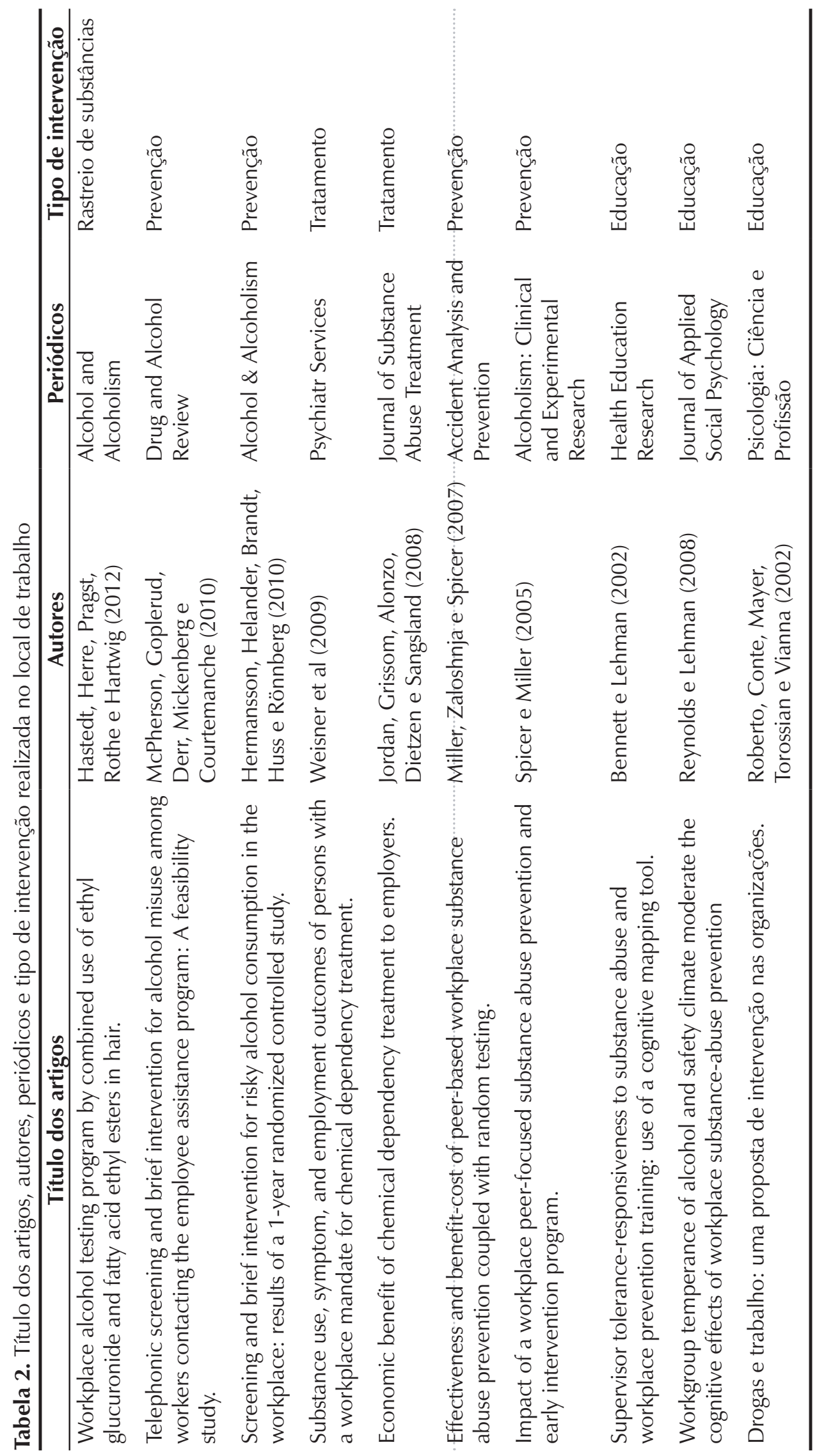


Quanto à autoria, todos os estudos tiveram sua autoria compartilhada por um ou mais autores. Os autores Rebecca S. Spicer e Ted R. Miller foram responsáveis pela publicação de dois artigos dessa análise que se referiram a trabalhos de prevenção ao uso de drogas no local de trabalho. O autor Wayne E. K. Lehman participou como autor de duas publicações referentes à educação do trabalhador sobre o uso de álcool e outras drogas. Os demais autores tiveram somente uma publicação.

Principais resultados e categorias

Diante da diversidade dos artigos, foi feita a Análise de Conteúdo a partir da separação dos artigos por categoria de pesquisa. As categorias que emergiram foram: 1 - Programas de prevenção; 2 - Programas de educação para álcool e outras drogas; 3 - Rastreio de drogas; 4 - Tratamento para dependentes.

1 - Programas de prevenção

Na categoria Programas de prevenção foram incluídos quatro artigos que se tratavam de programas de prevenção desenvolvidos no ambiente de trabalho para usuários de álcool e outras drogas. Dois dos quatro artigos tinham como objetivo avaliar a eficácia da intervenção breve (IB) no ambiente de trabalho para o uso de álcool. Os outros dois artigos tratavam-se da avaliação do programa de pares como estratégia de prevenção ao uso de álcool. Os quatro artigos sobre programas de prevenção serão descritos a seguir.

O artigo de McPherson et al. (2010) utilizou a modalidade da triagem de sujeitos via telefone. Os autores testaram a viabilidade da implantação de uma triagem telefônica com o uso do Alcohol Disorders Identification Test (AUDIT) em um call center no qual também eram oferecidos aos funcionários uma IB motivacional, encaminhamentos e follow-up. Segundo esses autores, foi possível a aplicação do SBIRT (Alcohol screening, brief intervention and referral to treatment) via telefone e como resultado 52\% dos trabalhadores tiveram redução no padrão de uso, passando de uso de risco ou moderado para problemas com o uso de álcool.

O artigo de Hermansson et al. (2010) é um estudo randomizado feito em uma empresa de grande porte do setor de transportes com o objetivo de avaliar a eficácia da IB quando associada com exames clínicos de saúde. Esse estudo utilizou grupo experimental e grupo controle com follow-up após 12 meses e relatou que a IB associada a exames clínicos de rotina foi efetiva para reduzir o consumo de álcool nesse intervalo de tempo.

O estudo de Spicer e Miller (2005) objetivou avaliar o impacto de um programa de prevenção por pares denominado Peercare nas taxas de acidentes de trabalho em uma empresa de grande porte. Para esses autores, o Peercare consiste em treinar colegas de trabalho para identificar e intervir quando um dos trabalhadores está com problemas relacionados ao uso de drogas, esse programa baseia-se na mudança de atitudes em relação ao uso de substâncias. Em 13 anos, a taxa de lesões no ambiente de trabalho reduziu $14 \%$ e, segundo esses autores, o programa de intervenções por pares reduz as lesões no contexto laboral. Esse estudo é longitudinal e oferece bons resultados decorrentes do programa de pares. Os pontos fortes desse estudo são o tempo de acompanhamento, que foi de 13 anos, e o tamanho da amostra, que se mostrou significativa.

Um ano depois, os mesmos autores, conjuntamente com Zaloshnja (Miller et al., 2007), avaliaram o custo e a efetividade de um programa Peercare associado a implementação de um programa de teste de drogas randomizado. Os autores observaram que os acidentes de trabalho declinaram com a aplicação do Peercare e apontam uma redução mais significativa quando o programa de pares foi aplicado conjuntamente com o teste de drogas randomizado. Segundo os autores, esses dois programas aplicados conjuntamente apresentaram bons resultados referentes a custo-benefício para os empregadores.

2 - Programas de educação no trabalho para álcool e outras drogas

Foram incluídos nessa categoria três artigos que tratavam do educar ou conscientizar os trabalhadores sobre o uso de álcool e outras drogas. Reynolds e Lehman (2008) utilizaram um treinamento de longa duração e também informações gerais sobre o uso de drogas no 
ambiente de trabalho. O método utilizado foi a comparação entre duas condições experimentais e um grupo controle. O grupo experimental 1 recebeu um treinamento de quatro semanas sobre as políticas internas e temas relacionados ao uso de drogas. $\mathrm{O}$ grupo experimental 2 recebeu duas horas de informação sobre o uso de drogas. Os resultados mostraram que havia um efeito significativo da interação entre o clima de segurança organizacional, normas de consumo e de formação de crenças. O treinamento de informações mostrou-se menos eficaz do que o treinamento intensivo.

Os estudos de Bennett e Lehman (2002) objetivaram compreender e melhorar a postura do supervisor em relação ao abuso de substâncias entre seus subordinados. Supervisores que receberam o treinamento foram mais propensos a melhorar em várias dimensões a capacidade de resposta do que os supervisores que receberam um treinamento mais didático, informativo ou em relação ao grupo controle.

Roberto et al. (2002) realizaram uma análise qualitativa sobre um programa educativo desenvolvido por uma consultoria. A metodologia utilizada para a intervenção foi a formação de grupos terapêuticos, entrevistas preliminares, oficinas, grupos operativos, planejamento estratégico, palestras, seminários e capacitações. A orientação terapêutica para esse trabalho foi a Psicanálise. Nesse trabalho foi desenvolvida uma política institucional específica, alicerçada na implantação de uma rotina norteadora para a abordagem dos funcionários quanto ao uso de drogas no trabalho; o credenciamento de locais para tratamento e a criação de comitês, liderados por funcionários, que passaram a ser responsáveis pela continuidade do programa após o término do trabalho da consultoria. Os resultados apontados pelos autores sugerem a importância da participação do maior número possível de funcionários, bem como a necessidade de criar uma política que considere os âmbitos administrativos, operacional e de saúde.

3 - Rastreio de drogas no local de trabalho

O trabalho de Hastedt et al. (2012) foi incluído na categoria Rastreio de drogas pois teve como objetivo ver a aplicabilidade dos testes Fatty acid ethyl esters (FAEEs) e do Ethyl glucuronide (ETG) no ambiente de trabalho. As amostras foram constituídas de cabelos de 78 trabalhadores que foram testados para o uso de álcool, sendo que o teste foi positivo para 58 deles. Quatro casos mostraram-se inconclusivos, pois indicavam incongruência nos marcadores. Os testes mostraram resultados efetivos para sua aplicação no ambiente de trabalho principalmente quando esses dois testes mencionados acima são aplicados conjuntamente.

\section{4 - Tratamento para dependentes}

Foram enquadrados nessa categoria dois artigos que se referiam a diferentes formas de tratamento para dependência química. No estudo de Weisner et al. (2009) foram comparados em dois grupos a influência de o funcionário possuir um encaminhamento ou mandato para seu tratamento de dependência química com funcionários que não receberam esse mandato. Os empregados que possuíam o mandato para o tratamento e os funcionários que não possuíam o mandato foram submetidos às mesmas formas de tratamento, com terapia de grupo, prevenção de recaída, terapia familiar e individual. Os resultados sugerem que os funcionários que possuíam um mandato para o tratamento tinham maior facilidade de chegar à abstinência e permaneciam por mais tempo em tratamento do que os trabalhadores que não possuíam o mandato.

Jordan et al. (2008) focalizaram seus estudos no custo-benefício gerado por um programa de tratamento para funcionários dependentes químicos. O tratamento incluía pelo menos um mês de internação e desintoxicação. Os resultados apontam que esses programas têm um bom custo-benefício para o empregador. Segundo os autores, houve reduções no absenteísmo, dos conflitos internos e melhora na produtividade.

Aspectos metodológicos dos artigos com foco em intervenções

Quanto aos aspectos metodológicos foram analisados o delineamento, a amostra, a população e os instrumentos de cada artigo. Nove artigos utilizaram a metodologia quantitativa e apenas o artigo escrito por Roberto et al. (2002) utilizou o método qualitativo. Quanto 
ao delineamento, seis artigos utilizaram o delineamento transversal e quatro utilizaram o longitudinal.

Os estudos de Hermansson et al. (2010), Weisner et al. (2009), Reynolds e Lehman (2008) e de Bennett e Lehman (2002) utilizaram a randomização com o objetivo de garantir uma melhor validade interna para as intervenções. Os autores McPherson et al. (2010) e Spicer e Miller (2005) utilizaram um pré-teste antes da intervenção e posteriormente foi realizado o seguimento, com pós-teste. O estudo de Hastedt et al. (2012) comparou o teste de cabelo com testes de sangue para garantir a validade dos resultados para sua pesquisa. Os estudos de Miller et al. (2007) e de Roberto et al. (2002) não relataram medidas de controle interno para as intervenções, pois se tratavam, respectivamente, de uma pesquisa de fonte documental e de uma pesquisa qualitativa.

Todas as populações dos estudos foram constituídas de trabalhadores sem especificação da categoria profissional. O tamanho da amostra variou de 78 a 2.021 trabalhadores. As análises dos dados variaram conforme os artigos. A Regressão linear foi utilizada em dois estudos, os demais artigos utilizaram análises estatísticas diferentes como: Qui-quadrado, Kruskal-Wallis, Regressão logística, Regressão dos quadrados mínimos, Poisson, Regressão binominal, Pearson, Anova, Manova, Análise de regressão hierárquica e Análise qualitativa.

Principais resultados e limitações dos artigos

Quanto aos resultados dos artigos, todos os dez estudos pesquisados apresentaram resultados positivos. Os estudos de McPherson et al. (2010) e Hermansson et al. (2010) mostraram efetividade da implantação da IB para o uso de álcool. Spicer e Miller (2005) e Miller et al. (2007) tiveram resultados positivos quanto às intervenções administradas no ambiente de trabalho com o programa de pares. Os autores Reynolds e Lehman (2008), Bennett e Lehman (2002) e Roberto et al. (2002) também mostraram que a conscientização e a educação para o uso de drogas podem ser importantes ferramentas no ambiente de trabalho para a prevenção do uso de álcool e outras drogas. Hastedt et al. (2012) mostraram que testes em cabelos para o rastreio de drogas podem ser efetivos para aplicação no ambiente de trabalho. Weisner et al. (2009) mostraram resultados positivos em trabalhadores que tinham mandato para o tratamento. Jordan et al. (2008) mostraram bom custo e efetividade de um programa de tratamento para usuários de drogas desenvolvido em uma empresa.

É importante ressaltar que apesar de os artigos mostrarem resultados positivos para as pesquisas, todos os trabalhos tiveram limitações em seus aspectos metodológicos. Reynolds e Lehman (2008) afirmaram não poder generalizar os resultados pelo fato de a intervenção ter sido feita somente em uma empresa. Também relatam que existiram variáveis externas que não foram controladas, como cultura organizacional, coesão grupal, entre outras. Spicer e Miller (2005) fizeram a pesquisa em três empresas do mesmo segmento empresarial, porém houve variáveis intervenientes como avanços tecnológicos e operacionais e aumento da consciência sobre o abuso de álcool e outras drogas.

Miller et al. (2007) citaram que não houve emparelhamento nos grupos em seu trabalho, o que pode ter contribuído para um viés de pesquisa. Weisner et al. (2009) mencionaram a impossibilidade da randomização dos grupos como limitação da pesquisa. Jordan et al. (2008) afirmam que seus estudos contêm ameaças à validade interna e não podem inferir que a melhora na produtividade e no absenteísmo se deu pela participação dos trabalhadores no tratamento oferecido. Hastedt et al. (2012) citam em seu trabalho que o rastreio de drogas é apenas uma parte de uma avaliação adequada com o objetivo de saber se o funcionário é ou não dependente de drogas. Deve-se considerar ainda que, por se encontrarem publicados, os dez artigos que foram incluídos nessa pesquisa têm maior chance de terem informações relativas a resultados significativos que apóiam a hipótese inicial dos estudos, retirando do universo de análise os resultados com hipótese nula ou os artigos com as hipóteses que não foram confirmadas.

\section{Discussão}

A revisão de literatura aponta que as intervenções feitas para o uso de substâncias psicoativas no ambiente de trabalho se enquadram em uma 
das quatro categorias que foram propostas neste artigo, sendo elas: Prevenção, Educação, Tratamento ou Rastreio de drogas, e que essa temática apresenta uma heterogeneidade de temas, apesar da escassez de estudos publicados.

Quanto ao idioma, os resultados demonstram a predominância das publicações em inglês e a carência de estudos com essa temática nas línguas espanhola e portuguesa. Também há uma carência de estudos randomizados principalmente quando se trata de intervenções realizadas no ambiente de trabalho. Houve o predomínio da análise quantitativa dos artigos publicados em inglês e o uso da análise qualitativa somente para o artigo publicado em português, o que pode ser explicado pelo tipo de objeto de estudo.

Todos os estudos utilizaram recortes transversais, em detrimento de metodologias longitudinais, que poderiam ser importantes para avaliação das consequências de longo prazo das intervenções realizadas. Estudos longitudinais seriam de grande importância para melhor compreender a eficácia das intervenções administradas no ambiente de trabalho, tanto da perspectiva organizacional como na perspectiva dos usuários de drogas.

Outra questão importante que se somou ao objetivo principal da pesquisa foi a forma da aplicação das intervenções. Quanto ao manejo dos programas, quando esses são associados a exames clínicos de rotina tornam-se mais aceitáveis no ambiente laboral, podendo ser aplicados conjuntamente com o médico ou enfermeiro do trabalho (Hermansson et al., 2010). Além disso, observou-se que quando a organização se engaja no tratamento do funcionário dependente de drogas esse responde melhor ao programa, ou seja, quando o funcionário é orientado ou possui um mandato para o tratamento, sua aderência ao programa é mais eficiente (Weisner et al., 2009). No estudo de Bennett e Lehman (2002) é salientada a importância do treinamento das lideranças para o trato com o funcionário usuário de drogas. Segundo esses autores, o supervisor treinado adquire maneiras mais eficazes de abordar a temática do uso de drogas. Ainda quanto ao manejo das intervenções, segundo Hastedt et al. (2012), deve-se prestar uma atenção maior em relação à ferramenta rastreio de drogas no ambiente de trabalho, já que essa abordagem envolve implicações éticas. Esse recurso é mais bem utilizado quando associado a outra forma de detecção do uso de drogas, como medidas de autorrelato, por exemplo.

Com relação ao modelo das intervenções estudadas, cabe destacar que não foi identificada uma única abordagem, embora algumas intervenções tenham utilizado estratégias semelhantes. Isso sugere que não existe um modelo pronto de intervenção a ser seguido. As empresas têm singularidades próprias e demandam tipos de intervenção diferentes decorrentes do clima organizacional e do tipo de instituição, assim como é mencionado no documento Brasil (2012). Os dez artigos analisados ofereceram resultados positivos quanto aos seus objetivos de pesquisa, porém não é possível afirmar qual método é mais eficaz no contexto de trabalho, já que não foram encontrados estudos que comparassem a eficácia das diferentes formas de intervenção. No que tange aos resultados da pesquisa, podemos afirmar que a Prevenção, a Educação para o álcool, o Tratamento e o Rastreio de drogas podem ser efetivos para produzir mudanças de percepção no ambiente de trabalho quanto ao uso de drogas, mas não se pode saber ao certo quais dessas intervenções oferecem resultados mais efetivos comparadas umas com as outras.

Devido à variabilidade dos métodos dos estudos avaliados não foi possível comparar as intervenções realizadas. As intervenções não apresentaram uma convergência de objetivos, instrumentos e população. Esse resultado aponta para inexistência de uma metodologia consolidada para mensurar as intervenções, dificultando a construção de replicações sistemáticas. Segundo a revisão elaborada por Webb et al. (2009), existem desafios consideráveis na construção de um corpo de trabalho com um foco contínuo para direcionar futuros empreendimentos. Os desafios estão relacionados às diferentes metodologias, à escolha da amostra, às restrições organizacionais, de lugar e de gênero, no comportamento de beber e escolha da unidade de alocação para a intervenção. 


\section{Considerações finais}

Com relação aos objetivos dessa revisão, foi possível identificar o que tem sido produzido na literatura sobre álcool e outras drogas no Brasil e em outros países referente a intervenções realizadas no contexto laboral. Somente $21,6 \%$ dos artigos tratava de intervenções, sendo que $10,3 \%$ se referia propriamente a intervenções realizadas no ambiente de trabalho para álcool e outras drogas, o que dificulta um direcionamento para futuras intervenções nesse campo.

Também foi possível investigar quais tipos de intervenção são mais utilizados no ambiente de trabalho. Em resposta a esses objetivos, o tema Prevenção apareceu com mais frequência quando se tratava de intervenções realizadas no contexto laboral. Apesar das limitações metodológicas dos artigos, a intervenção breve e o programa de pares se mostraram programas que podem ser usados para embasar uma nova forma de trabalho pautada na prevenção ao uso de drogas nas empresas, contribuindo para a reflexão da prática dos profissionais de Recursos Humanos, mais embasadas na promoção da saúde.

Outras categorias de intervenções investigadas como a Educação para o uso de drogas, o Tratamento para dependentes e o Rastreio de drogas devem ser empregadas de modo que permitam uma reflexão crítica das práticas dos psicólogos, levando ao questionamento dos modos de pensar e atuar nas organizações e permitindo um olhar diferenciado sobre a saúde do trabalhador em relação ao tema uso de álcool e outras substâncias.

Constata-se que ainda não foram encontrados estudos que relatem a eficácia de intervenções no contexto de trabalho brasileiro na área de drogas, indicando a necessidade de novas frentes de pesquisa no Brasil. Conclui-se que o conjunto de estudos avaliados, mesmo em outros países, ainda é insuficiente de evidências científicas e demanda mais estudos que busquem investigar a validade dessas intervenções e sua aplicabilidade no contexto de trabalho. Nessa perspectiva, destaca-se a importância de que novas pesquisas sejam conduzidas com o objetivo de identificar quais variáveis contribuem mais significativamente para o desfecho da intervenção, para que elas possam ser priorizadas no contexto laboral.

\section{Limitações}

Este estudo apresentou como limitações a não inclusão de todas as bases de dados existentes e de artigos publicados em outras línguas que não a inglesa, a espanhola e a portuguesa. Além disso, a técnica de vocabulário controlado pode ter restringido os trabalhos pesquisados, mas, por outro lado, ofereceu maior sistematização e possibilitou a replicação dos resultados. 


\section{Maira Leon Ferreira}

Mestre em Psicologia pela Universidade Federal de Juiz de Fora, Juiz de Fora - MG. Brasil. E-mail:mleonferreira@yahoo.com.br

\section{Laisa Marcorela Andreoli Sartes}

Docente de Graduação e de Pós graduação do Departamento de Psicologia, Instituto de Ciências Humanas, Universidade Federal de Juiz de Fora, Juiz de Fora - MG. Brasil.

E-mail: laisa.sartes@ufjf.edu.br

\section{Endereço para envio de correspondência:}

Universidade Federal de Juiz de Fora, Instituto de Ciências Humanas, Departamento de Psicologia. Rua José Lourenço Kelmer, s/n, Instituto de Ciências Humanas São Pedro. CEP 36036-900 - Juiz de Fora, MG - Brasil.

Recebido 27/08/2013, Aprovado 13/10/2013. 
Beck, L. M., \& David, H. M. S. L. (2007). O abuso de drogas e o mundo do trabalho: possibilidades de atuação para o enfermeiro. Escola Anna Nery, 11(4), 706-711. http://dx.doi.org/10.1590/ S1414-81452007000400024.

Bennett, J. B., \& Lehman, W. E. (2002). Supervisor tolerance-responsiveness to substance abuse and workplace prevention training: use of a cognitive mapping tool. Health Education Research, 17(1), 27-42. http://dx.doi.org/10.1093/ her/17.1.27. PMid:11890175

Brasil (2012). Prevenção ao uso de álcool e outras drogas no ambiente de trabalho: conhecer para ajudar. Brasília: Secretaria Nacional Antidrogas/ Serviço Social da Indústria. Recuperado em 19 de março de 2015, de http://pt.scribd.com/ doc/116946566/Prevencao-do-uso-de-alcool-eoutras-drogas-no-ambiente-de-trabalho\#scribd

Hastedt, M., Herre, S., Pragst, F., Rothe, M., \& Hartwig, S. (2012). Workplace alcohol testing program by combined use of ethyl glucuronide and fatty acid ethyl esters in hair. Alcohol and Alcoholism (Oxford, Oxfordshire), 47(2), 127132. http://dx.doi.org/10.1093/alcalc/agr148. PMid:22162917

Hermansson, U., Helander, A., Brandt, L., Huss, A., \& Rönnberg, S. (2010). Screening and brief intervention for risky alcohol consumption in the workplace: results of a 1-year randomized controlled study. Alcohol and Alcoholism (Oxford, Oxfordshire), 45(3), 252-257. http://dx.doi. org/10.1093/alcalc/agq021. PMid:20406791

Jordan, N., Grissom, G., Alonzo, G., Dietzen, L., \& Sangsland, S. (2008). Economic benefit of chemical dependency treatment to employers. Journal of Substance Abuse Treatment, 34(3), 311-319. http://dx.doi.org/10.1016/j. jsat.2007.05.001. PMid:17614238

Mangione, T. W., Howland, J., Amick, B., Cote, J., Lee, M., Bell, N. et al. (1999). Employee drinking practices and work performance. Journal of Studies on Alcohol, 60(2), 261-270. http://dx.doi.org/10.15288/jsa.1999.60.261. PMid:10091965

McPherson, T. L., Goplerud, E., Derr, D., Mickenberg, J., \& Courtemanche, S. (2010). Telephonic screening and brief intervention for alcohol misuse among workers contacting the employee assistance program: A feasibility study. Drug and Alcohol Review, 29(6), 641-646. http:// dx.doi.org/10.1111/j.1465-3362.2010.00249.x. PMid:20973849
Mendonça, G. G., Souza, N. P., \& Oliani, S. M. (2008). Assédio moral: práticas coercitivas nas relações de trabalho. Revista Eletrônica de Ciências Empresariais, 1(2), 1-9. Recuperado em 19 de março de 2015, de http://web.unifil. br/docs/empresarial/2/2.pdf

Miller, T. R., Zaloshnja, E., \& Spicer, R. S. (2007). Effectiveness and benefit-cost of peer-based workplace substance abuse prevention coupled with random testing. Accident; Analysis and Prevention, 39(3), 565-573. http://dx.doi. org/10.1016/j.aap.2006.10.001. PMid:17125723

National Institute on Alcohol Abuse and Alcoholism - NIAAA (1997). Ninth special report to the U.S. Congress on alcohol and health. Bethesda, MD: US Department of Health and Human Services. Recuperado em 19 de março de 2015, do NIAAA (National Institute on Alcohol Abuse and Alcoholism): http://www.niaaa.nih. gov/publications

Newcomb, M. D. (1988). Drug use in the workplace: risk factors for disruptive substance use among young adults. Dover, MA: Auburn House.

Normand, J., \& Salyards, S. (1989). An empirical evaluation of preemployment drug testing in the United States Postal Service: interim report of findings. NIDA Research Monograph, 91, 111-138. PMid:2509921.

Organização Mundial da Saúde - OMS. (1993). Classificação de transtornos mentais e de comportamento da CID 10. Porto Alegre: Artmed.

Reynolds, G. S., \& Lehman, E. K. (2008). Workgroup temperance of alcohol and safety climate moderate the cognitive effects of workplace substance-abuse prevention. Journal of Applied Social Psychology, 38(7), 1827-1866. http:// dx.doi.org/10.1111/j.1559-1816.2008.00371.x.

Roberto, C. S., Conte, M., Mayer, R. T., Torossian, S. T., \& Vianna T. R. (2002). Drogas e trabalho: uma proposta de intervenção nas organizações. Psicologia: Ciência e Profissão, 22(1), 18-29. http://dx.doi.org/10.1590/S141498932002000100004.

Roman, P. M., \& Blum, T. C. (1996). Alcohol: a review of the impact of worksite interventions on health and behavioral outcomes. American Journal of Health Promotion, 11(2), 136-149. http://dx.doi.org/10.4278/0890-1171-11.2.136. PMid:10163599 
Spicer, R. S., \& Miller, T. R. (2005). Impact of a workplace peer-focused substance abuse prevention and early intervention program. Alcoholism, Clinical and Experimental Research, 29(4), 609-611. http://dx.doi.org/10.1097/01. ALC.0000158831.43241.B4. PMid:15834226

Spricigo, J. N., \& Alencastre, M. B. (2004). O enfermeiro de Unidade Básica de Saúde e o usuário de drogas - um estudo em BiguaçúSC. Revista Latino-Americana de Enfermagem, 12(spe), 427-432. http://dx.doi.org/10.1590/ S0104-11692004000700019.

Vergara, S. C. (2005). Métodos de pesquisa em administração. São Paulo: Atlas.
Webb, G., Shakeshaft, A., Sanson-Fisher, R., \& Havard, A. (2009). A systematic review of work-place interventions for alcohol-related problems. Addiction (Abingdon, England), 104(3), 365-377. http://dx.doi.org/10.1111/j.13600443.2008.02472.x. PMid:19207344

Weisner, C., Lu, Y., Hinman, A., Monahan, J., Bonnie, R. J., Moore, C. D. et al. (2009). Substance use, symptom, and employment outcomes of persons with a workplace mandate for chemical dependency treatment. Psychiatric Services (Washington, D.C.), 60(5), 646-654. http://dx.doi.org/10.1176/ps.2009.60.5.646. PMid:19411353 